\title{
The Significance of Augmented High-Grade Squamous Intraepithelial Lesion Detection on Pap Test Examination: Partial Results from the RODEO Study Team
}

\author{
Cristovam Scapulatempo ${ }^{a}$ José Humberto T.G. Fregnani ${ }^{a, b}$ Natália Campacci ${ }^{a}$ \\ Júlio Cesar Possati-Resende ${ }^{a}$ Adhemar Longatto-Filho ${ }^{\text {b-e }}$ The RODEO Study Team \\ a Barretos Cancer Hospital, Pio XII Foundation, b Molecular Oncology Research Center, Barretos Cancer Hospital, \\ Pio XII Foundation, Barretos, and ' Laboratory of Medical Investigation 14, Faculty of Medicine, University of São Paulo,

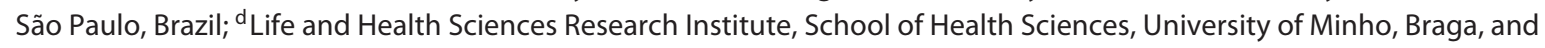 \\ e Life and Health Sciences Research Institute/3B's - PT Government Associate Laboratory, Guimarães, Portugal
}

\section{Key Words}

Pap test · Cytological screening · Liquid-based cytology · SurePath ${ }^{\mathrm{TM}}$. Cytopathology

\begin{abstract}
Objective: This study sought to ascertain the significance of augmented high-grade squamous intraepithelial lesion (HSIL) detection by Pap test using both conventional smear and liquid-based cytology (LBC) in a high-risk population. Study Design: We conducted a direct-to-vial study to compare the diagnostic performance of Pap smear versus LBC in a high-risk population of women referred for colposcopy at a gynecologic ambulatory clinic at the Barretos Cancer Hospital in Brazil during 2011. Results: The detection of both low-grade squamous intraepithelial lesions (LSILs) and HSILs was significantly greater $(p=0.04$ and $p=0.033$, respectively) in the LBC arm [84 LSIL cases (5.7\%) and $148 \mathrm{HSIL}$ cases $(10.1 \%)]$ than in the conventional smear arm [66 LSIL cases (4.1\%) and $126 \mathrm{HSIL}$ cases (7.9\%)]; however, no differences were found for invasive squamous carcinoma or adenocarcinoma ( $p=0.678)$. Of 3,071 women who were examined cytologically $(1,604$ conventional preparations and 1,467 LBC)
\end{abstract}

and colposcopically, biopsies were available for 279 conventional preparations (17.6\%) and 325 LBC preparations (22.2\%). No significant differences were found between the methods with respect to diagnostic performance. Conclusion: $\mathrm{LBC}$ was significantly superior to conventional smears for the detection of LSILs and HSILs, but these results did not influence biopsy confirmation. Both methods showed similar performances with high positive predictive values but very low sensitivities.

(c) 2013 S. Karger AG, Basel

\section{Introduction}

The impassioned disputes between defenders of conventional Pap smears and those who believe in the supremacy of liquid-based cytology (LBC) are generally

The RODEO Study Team is based at the Barretos Cancer Hospital and includes the following members: Teóclito Saccheto, Maíra Stein, Fábio de Paula Mateus, Eduardo Tadeu da Silva, Michele M. Castro Alves, Fábio Cardoso de Lima, Erlaine Martins Suriano and Edmundo C. Mauad C.S. and J.H.T.G.F. contributed equally to this work.

\section{KARGER}

E-Mail karger@karger.com www.karger.com/acy
(C) 2013 S. Karger AG, Basel

0001-5547/13/0575-0489\$38.00/0
Correspondence to: Dr. Adhemar Longatto-Filho

Laboratory of Medical Investigation 14

Faculty of Medicine, University of São Paulo

1246-903 São Paulo (Brazil)

E-Mail longatto16@ hotmail.com 
supported by the belief that one of these methods has the potential to detect more cases of high-grade squamous intraepithelial lesions (HSILs) on routine screenings for cervical cancer even in high-risk settings [1]. Moreover, LBC is believed to improve the quality of sample preparations, be more cost-effective, allow the use of computer-assisted screening and provide residual material for ancillary testing, including human papillomavirus (HPV) testing, all of which strongly favor the use of LBC [2-4].

To date, the routine method of cervical cancer screening supported by the Federal Government in Brazil is based on the conventional Papanicolaou (Pap) test. Unfortunately, despite many efforts, the high incidence and high mortality rate of cervical cancer have not been adequately addressed, especially in poor regions [5]. The conventional Pap test is a sophisticated methodology that requires a high degree of refinement when collecting, preparing and fixing the cervical samples [6]. The reputation for simplicity of the conventional Pap test has been shown to be undeserved; technicians should be regularly trained, the quality control must be rigorous, and the number of slides examined each day must be limited for the method to be minimally efficient. Consequently, the price of this complex test is not insignificant $[6,7]$. Therefore, new methodologies are currently being advocated to overcome the limitations of conventional Pap tests, increase the detection of cervical lesions and reduce mortality [7]. LBC appears to be an attractive option to replace the conventional method because it preserves cervical samples immediately after collection in an LBC vial, which provides a better preparation and a presumed superior cytological evaluation [3]. In addition, LBC is a realistic option for computer-assisted analysis, molecular testing and HPV detection [5, 7].

The RODEO study was designed to evaluate 30,000 consecutive women at the Barretos Cancer Hospital (Barretos, Brazil), the Leonor Mendes de Barros Hospital and the Hospital das Clínicas of the Faculty of Medicine of the São Paulo State University (São Paulo, Brazil). The principal goal of our results is to apply them in an algorithm to prevent and control cervical cancer. The RODEO study was implemented at the Barretos Cancer Hospital to provide a realistic basis for the integration of $\mathrm{LBC}$, automated screening and the inclusion of HPV DNA testing in the screening routine. This study reports on the performance of $L B C$ versus conventional preparations in a high-risk setting and discusses the significance of increased detection of cervical lesions using manual screening conditions.

\section{Materials and Methods}

\section{Study Design}

This study represents a part of the RODEO study that was performed exclusively at the Barretos Cancer Hospital. The study was a direct-to-vial study, and the samples were collected in the Gynecologic Oncology Ambulatory Clinic after women were referred due to abnormal Pap tests, for treatment to control cervical lesions and for other reasons.

Only women whose Pap tests were carried out in the Gynecologic Oncology Department were included. Pregnant women were not enrolled in the study because endocervical sampling is contraindicated for them. Samples were collected in 4 ambulatory clinics, and the women were randomly assigned to receive LBC or a conventional smear. These women were referred to the ambulatory clinics for various reasons, including follow-up after treatment for cancer of the cervix, vulva, ovaries and endometrium, among others. Because of their medical histories, these women were considered high risk for HPV-induced diseases.

Preparation of Cytological Samples and Diagnostic Classification

Conventional Pap test samples were collected using an Ayre's wooden spatula to scrape the ectocervix and a Combi Cervex ${ }^{\mathrm{TM}}$ brush (Rover, The Netherlands) to scrape the endocervix. Only a single slide was prepared for each sample. The samples were smeared on a slide and immediately fixed with a polyethylene glycol fixative spray. LBC samples were collected using a Combi Cervex brush (Rover), placed into a plastic vial with SurePath ${ }^{\mathrm{TM}}$ liquid medium (Tripath Imaging Inc., Burlington, N.C., USA) and prepared according to the manufacturer's instructions. The classification of the cytological alterations was performed according to the Bethesda 2001 system [8].

\section{Statistical Analysis}

The statistical data were analyzed using SPSS for Windows ${ }^{\circledR}$ version 20.0 (SPSS Inc., Chicago, Ill., USA). A t test was used to compare the women's ages. The prevalence rates were compared using a $\mathrm{z}$ approximation. Confidence intervals for the proportions were also calculated. The significance level was set at $5 \%$. Cervical biopsy [cervical intraepithelial neoplasia (CIN) 1+, CIN2+] was considered the gold standard for calculating the sensitivity, specificity and predictive values of the conventional and LBC methods. Analysis of the area under the curve (AUC) of a receiver operating characteristic curve was used to compare the diagnostic performance of each cytology method in the different settings.

Ethics

All women signed an informed consent document before being included in the study. The study protocol was approved by the local Ethics Committee of the Barretos Cancer Hospital (No. 244/2009).

\section{Results}

A total of 4,726 women were included in the study; of these, 2,378 women (50.3\%) underwent conventional smears and 2,348 women (49.7\%) underwent LBC. The 
Table 1. Distribution of cases according to the result and type of cervical cytology

\begin{tabular}{lcccc}
\hline Result of cervical cytology & $\begin{array}{l}\text { Conventional cytology } \\
(\mathrm{n}=1,604)\end{array}$ & LBC $(\mathrm{n}=1,467)$ & $\begin{array}{l}\text { Prevalence } \\
\text { rate }\end{array}$ & $\mathrm{p}$ value \\
\hline Negative & $1,267(79.0 \%)$ & $1,090(74.3 \%)$ & 0.94 & 0.002 \\
ASC-US & $58(3.6 \%)$ & $50(3.4 \%)$ & 0.94 & 0.763 \\
ASC-H & $60(3.7 \%)$ & $68(4.6 \%)$ & 1.24 & 0.211 \\
AGC & $5(0.3 \%)$ & $5(0.3 \%)$ & 1.00 & 1.000 \\
LSIL & $66(4.1 \%)$ & $84(5.7 \%)$ & 1.39 & 0.040 \\
HSIL & $126(7.9 \%)$ & $148(10.1 \%)$ & 1.28 & 0.033 \\
Invasive carcinoma (SCC/AD) & $6(0.4 \%)$ & $7(0.5 \%)$ & 1.25 & 0.678 \\
Unsatisfactory & $16(1.0 \%)$ & $15(1.0 \%)$ & 1.02 & 0.949 \\
\hline
\end{tabular}

$\mathrm{p}$ values were calculated using $\mathrm{z}$ approximation. ASC-US = Atypical squamous cells of undetermined significance; ASC-H = atypical squamous cells - cannot exclude HSIL; AGC = atypical glandular cells; SCC = squamous cell carcinoma; $\mathrm{AD}=$ adenocarcinoma.

mean age for the Pap smear group was 45.6 years, and the mean age for the LBC group was 48.6 years ( $t$ test: $p<$ 0.001 ). Abnormal Pap test results were detected in 390 conventional smears $(16.4 \%)$ and 514 LBCs $(22.2 \%$; $<<0.001)$.

For diagnostic performance analyses comparing conventional smears with LBC, patients with a history of pelvic radiotherapy were excluded from the study. Therefore, 1,604 Pap smears and 1,467 LBCs were included in this portion of the study.

Table 1 shows the distribution of the cytological results and the overall distribution following the cytological examinations. Abnormal cases were identified according to their cytological classification, and these specific cytological alterations showed significant differences between the LBC and conventional smears overall $(\mathrm{p}<0.001)$. The main differences between LBC and conventional smears, respectively, included the following: low-grade squamous intraepithelial lesion (LSIL), 84 cases (5.7\%) vs. 66 cases (4.1\%; $\mathrm{p}=0.040)$, and HSIL, 148 cases $(10.1 \%)$ vs. 126 cases $(7.9 \%$; $\mathrm{p}=0.033)$. There was no difference in the unsatisfactory result rates between $\mathrm{LBC}$ and conventional smears.

Table 2 extends the data from table 1 and correlates the cytological diagnoses with the biopsy results. Table 3 shows the performance in diagnosing CIN1+ and CIN2+ according to the type of cytology and the cytological criterion (ASC-H+, LSIL+, HSIL+). Both of the methods performed similarly despite the significantly higher number of LSILs and HSILs detected by LBC. Using biopsyproven $\mathrm{CIN} 1+$ as a cutoff, no significant differences were observed between the methods. However, using CIN2+ as a cutoff showed a slightly favorable result for conventional smears when calculated for the ASC-H+ or LSIL+ cytological criterion.

Increased HSIL Detection on LBC
Table 2. Number and percentage of cases with cervical biopsy data according to the cervical cytology result

\begin{tabular}{lllr}
\hline Type of cytology & \multicolumn{3}{l}{ Biopsy } \\
\cline { 2 - 4 } & negative & CIN1+ & CIN2/3+ \\
\hline $\begin{array}{l}\text { Conventional } \\
\text { LSIL+ }\end{array}$ & $13(22.4 \%)$ & $20(34.5 \%)$ & $25(43.1 \%)$ \\
HSIL+ & $15(12.0 \%)$ & $13(10.4 \%)$ & $97(77.6 \%)$ \\
LBC & $19(25.0 \%)$ & $27(35.5 \%)$ & $30(39.5 \%)$ \\
$\quad$ LSIL+ & $23(15.6 \%)$ & $8(5.4 \%)$ & $116(78.9 \%)$ \\
HSIL+ & & & \\
\hline
\end{tabular}

Interestingly, for both methods, the sensitivity and negative predictive values were higher if ASC-H+ was adopted as the cutoff, and the values diminished progressively when LSIL+ and HSIL+ were used as the cutoffs. Conversely, the specificity and positive predictive values were lower for an ASC-H+ cutoff and rose progressively for LSIL+ and HSIL+ cutoffs.

Higher AUCs were achieved when HSIL+ was used as the cytological criterion to diagnose HSILs. In this setting, there was no difference in the diagnostic performance between LBC and conventional smears. The respective AUCs were 0.686 and $0.712(\mathrm{p}=0.482)$.

\section{Discussion}

Methodological improvements in gynecological cytology represent essential tools that should be incorporated into programs for cancer prevention because cytotechnologists need a more robust and adequate armamentar- 
Table 3. Performance of conventional cytology and LBC in diagnosing CIN1+ and CIN2+

\begin{tabular}{lllllllll}
\hline $\begin{array}{l}\text { Gold } \\
\text { standard } \\
\text { (biopsy) }\end{array}$ & $\begin{array}{l}\text { Cyto- } \\
\text { logical } \\
\text { criterion }\end{array}$ & $\begin{array}{l}\text { Type of } \\
\text { cytology }\end{array}$ & Sensitivity, $\%$ & Specificity, $\%$ & Positive PV, \% & Negative PV, \% & AUC & $\begin{array}{l}\text { p } \\
\text { value }\end{array}$ \\
\hline CIN1+ & ASC-H+ conventional & $90.9(86.1-94.4)$ & $28.2(18.1-40.1)$ & $78.8(73.0-83.7)$ & $51.3(34.8-67.6)$ & $0.595(0.535-0.653)$ & 0.305 \\
& & LBC & $91.6(87.4-94.8)$ & $19.8(12.0-29.8)$ & $76.0(70.7-80.9)$ & $45.9(29.5-63.1)$ & $0.557(0.501-0.612)$ \\
& LSIL+ & conventional & $75.0(68.5-80.7)$ & $62.0(49.7-73.2)$ & $85.2(79.3-90.0)$ & $45.8(35.6-56.3)$ & $0.685(0.627-0.739)$ & 0.254 \\
& & LBC & $75.7(69.8-81.0)$ & $51.2(40.1-62.1)$ & $81.2(75.4-86.1)$ & $43.1(33.4-53.3)$ & $0.634(0.580-0.687)$ \\
& HSIL+ & conventional & $53.4(46.3-60.3)$ & $80.3(69.1-88.8)$ & $88.8(81.9-93.7)$ & $37.0(29.4-45.2)$ & $0.668(0.610-0.723)$ & 0.309 \\
& & LBC & $51.9(45.3-58.4)$ & $73.3(62.6-82.2)$ & $84.4(77.5-89.8)$ & $35.4(28.4-42.9)$ & $0.626(0.571-0.678)$ \\
\hline CIN2++ & ASC-H+ conventional & $96.0(91.6-98.5)$ & $25.8(18.5-34.3)$ & $60.4(53.9-66.6)$ & $84.6(69.5-94.1)$ & $0.609(0.549-0.667)$ & 0.021 \\
& & LBC & $92.2(87.4-95.6)$ & $16.5(10.7-24.0)$ & $61.5(55.6-67.1)$ & $59.5(42.1-75.2)$ & $0.544(0.488-0.599)$ \\
& LSIL+ & conventional & $80.8(73.6-86.7)$ & $52.3(43.3-61.2)$ & $66.7(59.3-73.4)$ & $69.8(59.6-78.7)$ & $0.666(0.607-0.721)$ & 0.049 \\
& & LBC & $76.0(69.4-81.9)$ & $42.1(33.6-51.0)$ & $65.5(58.8-71.7)$ & $54.9(44.7-64.8)$ & $0.591(0.535-0.645)$ \\
& HSIL+ & conventional & $64.2(56.0-71.9)$ & $78.1(70.0-84.9)$ & $77.6(69.3-84.6)$ & $64.9(56.8-72.4)$ & $0.712(0.655-0.764)$ & 0.482 \\
& & LBC & $60.4(53.1-67.4)$ & $76.7(68.6-83.6)$ & $78.9(71.4-85.2)$ & $57.3(49.7-64.7)$ & $0.686(0.632-0.736)$ \\
\hline
\end{tabular}
HSIL.

Figures in parentheses represent 95\% confidence intervals. PV = Predictive value; ASC-H = atypical squamous cells - cannot exclude

ium to detect cervical alterations (mainly HSILs) than is provided by the conventional cytological smear. The diagnostic performance calculations for both of the methods we tested followed very stringent parameters, which certainly repressed the positive impact of LBC performance, even though almost $100 \%$ more HSILs were detected by LBC compared to conventional smear preparations. The conventional Pap test, even when performed in a controlled setting, has important limitations when identifying cervical lesions and has not infrequently demonstrated inferior performance compared with LBC in reports from developed countries [9]. However, when LBC and conventional smears have been measured against a biopsyproven result, the differences have not shown significant improvements in terms of ameliorated performance in other reports from developed countries [10]. However, this assumption is not necessarily true for poorer settings [1]. The reasons for these discrepancies could be attributed to several factors, and most of those factors have been discussed previously. The level of experience of the cytotechnicians and favorable conditions for screening are critical for Pap test performance [11]. Moreover, conventional smears largely depend on the expertise of welltrained nurses and medical doctors to collect the samples, smear them onto slides and fix them properly. Professionals with high levels of expertise certainly represent an important variable for the success of cancer prevention programs, but even under the good conditions that we have at Barretos Cancer Hospital, we observed that convention- al cytology identified significantly fewer cytological abnormalities than LBC. The real meaning of the higher detection rate for LSILs and HSILs in our series is a challenging point that should be evaluated seriously. Firstly, the Pap test is recognized as an excellent method in terms of its specificity, which is usually very high [11-14]. Accordingly, if a Pap test result is categorized as an HSIL, the result is likely to be correct. In addition, even without colposcopic evidence of a cervical lesion, it is prudent to carefully follow women diagnosed with an HSIL. This recommendation is most likely the most disputable issue regarding the real meaning of the superior LBC performance. Well-conducted meta-analyses based on reports from developed countries have systematically demonstrated that LBC and conventional cytological preparations perform similarly to detect HSILs $[9,10]$. However, a number of studies have described better performance by LBC in high-risk settings to detect high-grade lesions and to decrease the unsatisfactory preparation rate [11-14].

The identification of cytological abnormalities appears to be favored in LBC samples because of the clean background and the homogenous and randomized distribution of normal and abnormal cells. Not surprisingly, the specificity of LBC has also been recognized to be higher than that of conventional smears [11]. Moreover, the superior performance of LBC to detect cervical lesions has been supported by previous studies in Brazil in populations at high risk for cervical cancer [1] but not in low-risk populations [10]. 
The AUC can be used to evaluate the most accurate model for diagnosing CIN; the higher the value of this area, the more accurate the model is in predicting CIN. To diagnose CIN1+, the largest AUC was found when LSIL+ was used as the cytological criterion. In this situation, both LBC and the conventional preparation had the same performance in diagnosing CIN1+. In the same manner, diagnosing CIN2+ using HSIL+ as the cytological criterion led to the highest AUCs, and both types of cytology had similar performances. Although these findings partially support the European meta-analysis [10], the better performance of LBC to detect HSILs in an assumed 'population with an increased risk of HPV-induced diseases' clearly obligated us to be confident with LBC and to carefully follow the women with HSILs that had not been confirmed by biopsy. The design of the current study presumed that in a population at low risk for HPV-induced lesions, there would be a lower prevalence of squamous intraepithelial lesions with supposed lower detection by cytology, which would be expected for postHPV vaccination women [15]; in contrast, high-risk populations should facilitate the identification of squamous intraepithelial lesions by cytology. However, with the number of cases we evaluated, biopsy confirmation should have been much greater to detect statistically relevant differences (data not shown).

Finally, LBC preparations are generally free of the biases related to conventional slides because both the sample preparation and staining procedures are automated. Even assuming a scenario in which LBC and conventional smears perform similarly, $\mathrm{LBC}$ is preferred due to the higher quality of the technical preparation and other potential inherent additional actions, including performing molecular HPV testing [16], creating a biological bank using the residual LBC material [17] and screening the results using computer-assisted systems [18]. The methodological superiority of LBC over conventional smears was clearly demonstrated several years ago by Hutchinson et al. [19], who identified nonhomogeneous cellular transfer from the sampling device to the slide as an important contributing factor that affected the efficacy of the conventional Pap smear; these authors observed that the abnormal cellularity varied significantly in 4 consecutive smears from the same woman. In contrast, LBC preparations maintained a constant diagnostic cellularity on each replicated preparation [19]. Recently, Kitchener et al. [20] provided robust arguments favoring LBC in the ARTISTIC trial, reporting an impressive study in which LBC performance was evaluated in detail compared with HPV testing. To initiate the trial, 10 laboratory members were exhaustively trained to read LBC slides and evaluate the time consumed in reading these preparations. This step was critical because the cytoscreener had to feel confident about the particular differences between conventional and LBC preparations [21]. This commentary is pertinent because our cytoscreeners and cytopathologists are very qualified professionals with a large amount of experience in reading conventional smears but not LBC. Despite the LBC training prior to the start of the study, the differences in familiarity between the 2 methodologies comprised several years and thousands of slides at the time the study started. This could explain, in part, the differences in the sensitivity and specificity indices between the RODEO and ARTISTIC trials; the former reported a sensitivity and specificity of 92.9 and $89.3 \%$, respectively, while the latter reported a sensitivity and specificity of 60.4 and $76.7 \%$, respectively.

Finally, our perception of LBC and its related facilities is that technological improvements in preparing cytological samples have contributed significantly to the enhancement of the detection of cytological abnormalities.

\section{Disclosure Statement}

This study was partially supported by $\mathrm{BD}$, which donated the SurePath vials and Papanicolaou staining reagents. The study design, statistical analyses, interpretation of the results and manuscript development were carried out by the team of the Barretos Cancer Hospital with no involvement from BD.

\section{References}

1 Longatto Filho A, Pereira SM, Di Loreto C, et al: DCS liquid-based system is more effective than conventional smears to diagnosis of cervical lesions: study in high-risk population with biopsy-based confirmation. Gynecol Oncol 2005;97:497-500.

2 Cox JT: Liquid-based cytology: evaluation of effectiveness, cost-effectiveness, and application to present practice. J Natl Compr Canc Netw 2004;2:597-611.

3 Strander B, Andersson-Ellström A, Milsom I, et al: Liquid-based cytology versus conventional Papanicolaou smear in an organized screening program: a prospective randomized study. Cancer 2007;111:285-291.

4 Levi AW, Chhieng DC, Schofield K, et al Implementation of FocalPoint GS locationguided imaging system: experience in a clinical setting. Cancer Cytopathol 2012;120:126133

5 Gamarra CJ, Valente JG, Silva GA: Correction for reported cervical cancer mortality data in Brazil, 1996-2005. Rev Saude Publica 2010; 44:629-638. 
6 Longatto-Filho A, Schmitt FC: Gynecological cytology: too old to be a pop star but too young to die. Diagn Cytopathol 2007;35:672673.

7 Longatto Filho A, Schmitt FC: Cytology education in the 21st century: living in the past or crossing the Rubicon? Acta Cytol 2010;54: 654-656.

8 Solomon D, Davey D, Kurman R, et al; Forum Group Members; Bethesda 2001 Workshop: The 2001 Bethesda System: terminology for reporting results of cervical cytology. JAMA 2002;287:2114-2119.

9 Davey E, Barratt A, Irwig L, et al: Effect of study design and quality on unsatisfactory rates, cytology classifications, and accuracy in liquid-based versus conventional cervical cytology: a systematic review. Lancet 2006;367: 122-132.

10 Arbyn M, Bergeron C, Klinkhamer P, et al: Liquid compared with conventional cervical cytology: a systematic review and meta-analysis. Obstet Gynecol 2008;111:167-177.

11 Longatto-Filho A, Maeda MY, Erzen M, et al: Conventional Pap smear and liquid-based cytology as screening tools in low-resource settings in Latin America: experience of the Latin American screening study. Acta Cytol 2005;49:500-506.
12 Siebers AG, Klinkhamer PJ, Grefte JM, et al: Comparison of liquid-based cytology with conventional cytology for detection of cervical cancer precursors: a randomized controlled trial. JAMA 2009;302:1757-1764.

13 Ronco G, Cuzick J, Pierotti P, et al: Accuracy of liquid based versus conventional cytology: overall results of new technologies for cervical cancer screening: randomised controlled trial. BMJ 2007;335:28.

14 Hovland S, Arbyn M, Lie AK, et al: A comprehensive evaluation of the accuracy of cervical pre-cancer detection methods in a high-risk area in East Congo. Br J Cancer 2010;102: 957-965.

15 Franco EL, Cuzick J: Cervical cancer screening following prophylactic human papillomavirus vaccination. Vaccine 2008;26(suppl 1):A16-A23.

16 Schmitt FC, Longatto-Filho A, Valent A, Vielh P: Molecular techniques in cytopathology practice. J Clin Pathol 2008;61:258267.
17 Arbyn M, Andersson K, Bergeron C, et al: Cervical cytology biobanks as a resource for molecular epidemiology. Methods Mol Biol 2011;675:279-298.

18 Wilbur DC, Black-Schaffer WS, Luff RD, et al: The Becton Dickinson FocalPoint GS Imaging System: clinical trials demonstrate significantly improved sensitivity for the detection of important cervical lesions. Am J Clin Pathol 2009;132:767-775.

19 Hutchinson ML, Isenstein LM, Goodman A, Hurley AA, Douglass KL, Mui KK, Patten FW, Zahniser DJ: Homogeneous sampling accounts for the increased diagnostic accuracy using the ThinPrep Processor. Am J Clin Pathol 1994;101:215-219.

20 Kitchener HC, Gilham C, Sargent A, et al: A comparison of HPV DNA testing and liquid based cytology over three rounds of primary cervical screening: extended follow up in the ARTISTIC trial. Eur J Cancer 2011;47:864871

21 Alves VA, Bibbo M, Schmitt FC, et al: Comparison of manual and automated methods of liquid-based cytology. A morphologic study. Acta Cytol 2004;48:187-193. 